\title{
ACM/Springer Mobile Networks and Applications (MONET) Special Issue on "Recent Advances in IEEE 802.11 WLANs: Protocols, Solutions and Future Directions"
}

\section{Editorial}

\author{
Periklis Chatzimisios • Yang Xiao • Ilenia Tinnirello • \\ Fabrizio Granelli • Ehab S. Elmallah
}

Published online: 3 April 2009

(C) Springer Science + Business Media, LLC 2009

With the rapid growth in popularity of wireless data services and the increasing demand for wireless connectivity, Wireless Local Area Networks (WLANs) have become more widespread and are making their way into commercial and public areas being almost everywhere including business, office and home deployments. WLANs based on the IEEE 802.11 standards enjoy an exceptionally high popularity owing to their simplicity of setup, configuration, and management, increased deployment flexibility, operability in

P. Chatzimisios $(\bowtie)$

Department of Informatics,

Alexander Technological Educational Institution,

P.O. BOX 141, GR-57400 Thessaloniki, Greece

e-mail: pchatzimisios@ieee.org

Y. Xiao

Department of Computer Science, University of Alabama,

101 Houser Hall, Box 870290, Tuscaloosa, AL 35487-0290, USA

e-mail: yangxiao@ieee.org

\section{Tinnirello}

Dipartimento di Ingegneria Elettrica,

Universita' degli Studi di Palermo,

Viale delle Scienze,

90128 Palermo, Italy

e-mail: ilenia.tinnirello@tti.unipa.it

F. Granelli

DISI, University of Trento,

Via Sommarive 14,

I-38050 Trento, Italy

e-mail: granelli@disi.unitn.it

\section{E. S. Elmallah}

Department of Computing Science, University of Alberta, Edmonton T6G 2E8 AB, Canada

e-mail: ehab@cs.ualberta.ca unlicensed frequency bands, low cost, and connectivity with minimal infrastructure changes.

Lately, the need for real-time multimedia services over WLANs to support applications such as Voice over IP (VoIP), audio/video (AV) streaming, Internet videoconferencing, IPTV, distance learning systems, entertainment and gaming programs has dramatically increased. The impressive proliferation of networking scenarios (e.g. multihop and mesh networks) and applications (e.g. real-time multimedia services) not natively supported by the original standard has boosted the design of several protocol extensions, which have been proposed and evaluated by the research community, the industry and the standardization groups.

However, despite recent advances in wireless technology, many research issues such as bandwidth management, power conservation, compatibility with legacy networks and error resilient design still need to be addressed. Furthermore, the dynamically-varying and error-prone nature of the wireless medium pose further challenges in providing efficient channel access mechanisms and Quality of Service (QoS) support over WLANs.

This special issue presents a collection of selected papers that represent advances towards the performance enhancement of IEEE 802.11 WLANs. A total of 37 high-quality papers were received from the open call and finally eight were selected to be published in this issue.

The first paper, "A Control Theoretic Approach for Throughput Optimization in IEEE 802.11e EDCA WLANs", by Patras, Banchs and Serrano, proposes a control theoretic approach to adapt the congestion window $(\mathrm{CW})$ to the conditions of the WLAN, based on an analytical model of its operation that is fully compliant with the IEEE 802.11e standard. The controller is a Proportional Integrator, able to 
perform a theoretic analysis to determine its configuration and to drive the WLAN to its optimal point of operation.

In the paper "Cross-Layer Scheduling with QoS Support over a Distributed Queuing MAC for Wireless LANs" Kartsakli, Alonso-Zárate, Alonso and Verikoukis propose four scheduling algorithms that are based on an efficient distributed MAC protocol named Distributed Queuing Collision Avoidance (DQCA). The proposed schemes combine the efficiency of opportunistic scheduling with the Quality of Service (QoS) provisioning through service differentiation in order to provide QoS guarantees. The authors study the throughput, delay and jitter performance of the proposed schemes through simulations for a scenario with heterogeneous traffic of voice, video, best-effort and background data traffic flows.

The paper "A Multi-Scale Statistical Control Process for Mobility and Interference Identification in IEEE 802.11" by Oliveira, Loureiro and Frery deals with the problem of estimating the wireless channel conditions in indoor environment. In fact, different optimization strategies can be pursued according to the physical origin (e.g. collisions or fading) of channel impairments. Thus, the availability of reliable estimation techniques for distinguishing different failure events has several practical implications. The authors present a new method for user centric management on IEEE $802.11 \mathrm{~b} / \mathrm{g}$ focused in improving the user experience.

The paper "DPS: An Architecture for VBR Scheduling in IEEE 802.11e HCCA Networks with Multiple Access Points", by Vergados, Vergados and Douligeris, is focused on the problem of interference management among overlapping polling-based networks. Since nowadays the unlicensed spectrum is overloaded, it is very likely that multiple overlapping networks work on the same channel, thus creating potential conflicts among polling commands and node transmissions, which are not managed via the carrier-sense function. The paper describes these problems and proposes an interesting multi-cell coordination scheme for coping with conflicting polling.

The paper "IEEE 802.11n MAC Enhancement and Performance Evaluation", by Wang and Wei, examines the network performance enhancement by the proposed IEEE 802.11n MAC layer features like aggregation, block acknowledgement and reverse direction mechanism. The authors implement a new 802.11n module in the NS-2 simulation platform and through simulations they demonstrate that IEEE 802.11n indeed improves the channel efficiency and provides high quality for VoIP services.
The paper "Packet Delay Metrics for IEEE 802.11 Distributed Coordination Function", by Raptis, Vitsas and Paparrizos, introduces a comprehensive packet delay analysis for wireless networks based on IEEE 802.11 Distributed Coordination Function (DCF). The approach is based on proper mathematical models that calculate a set of packet delay metrics, including average delay, delay distribution and jitter. The developed models are based on calculating station's delay time at the transmission slot(s) plus the average time that station defers at backoff slots before successful transmission, leading to simple and computationally efficient models.

In the paper "Performance Study of A Mobile Multi-Hop 802.11a/b Railway Network using Passive Measurement", Zhou, Sharif, Hempel, Mahasukhon, Wang and Chen build an outdoor multi-hop multi-interface railroad testbed and study the performance of IEEE $802.11 \mathrm{a} / \mathrm{b}$ protocols in a large-scale mobile railway network. They introduce a largescale passive measurement approach that enables a global performance view for the entire monitored network. Furthermore, the paper discusses the implications on guaranteeing the quality of mobile services in IEEE 802.11-family railway networks.

In the paper "Green WLANs: On-demand WLAN Infrastructure", Jardosh, Papagiannaki, Belding, Almeroth, Iannaccone and Vinnakota consider achieving energy conservation in dense WLAN deployments where a potentially large number of access points with overlapping coverage areas can be managed centrally. The paper presents resource on-demand (RoD) strategies for switching power to the access points to reduce the total energy consumption while maintaining coverage to all users. The authors also discuss several future research directions towards the wide-spread deployment of RoD WLANs.

We would like to thank all authors for submitting their work to this Special Issue and the external reviewers for their tireless work and efforts during the reviewing process. We also wish to thank MONET Editorial Staff for their continuous support and professionalism. Finally, we want to express our gratitude to the Editor-in-Chief, Professor Imrich Chlamtac, for giving us this great opportunity of organizing this special issue and for his invaluable support and suggestions.

We are most pleased to present this issue and we hope that it will provide a good source for up-to-date information on this exciting topic.

Enjoy the issue! 


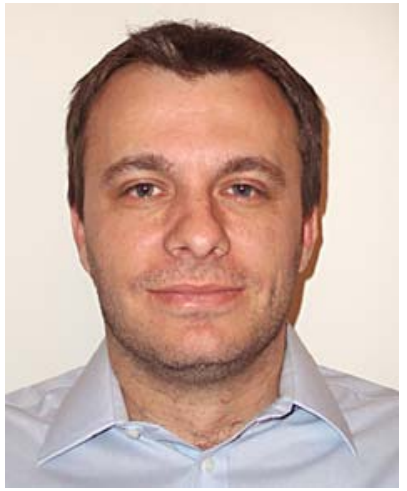

Periklis Chatzimisios received his BSc degree in Informatics from Technological Educational Institution of Thessaloniki, Greece, in 2000 and his $\mathrm{PhD}$ degree in wireless communications from Bournemouth University, U.K., in 2005. He is currently working as a Postdoctoral Researcher and adjunct Assistant Professor with the Department of Informatics at the Technological Educational Institution of Thessaloniki. He has been also employed as an adjunct Lecturer by University of Macedonia, Aristotle University of Thessaloniki and University of Western Macedonia, Greece, where he is engaged with teaching and research on computer and telecommunications networks. $\mathrm{He}$ is also collaborates with the Hellenic Open University (EAP). Dr. Chatzimisios serves on the Editorial Board for the IEEE Communications Surveys \& Tutorials and the International Journal of Computers and Applications. He is the Technical Program Co-Chair for the 1st International Conference on Mobile Lightweight Wireless Systems (MOBILIGHT 2009), the Publicity Co-Chair for the IEEE International Symposium on Computers and Communications Conference (ISCC 2009) and the Steering Committee Chair for the 4th Workshop on multiMedia Applications over Wireless Networks (MediaWiN 2009). In the past he has served as the Publication CoChair and National Ambassador of Greece for the IEEE International Symposium on Personal, Indoor and Mobile Radio Communications (PIMRC 2008) and the General Co-Chair for MediaWiN 2006, 2007 and 2008 Workshops. He has published more than 35 peer-reviewed papers in international journals, books and conferences related to the research area of wireless networks and in particular on performance modelling, analysis and enhancement, discrete-event simulation and multimedia communications. Dr. Chatzimisios's research has been supported by a research fellowship from the Greek State Scholarship Foundation (I.K.Y.) and his published research work has received more than 300 citations by other researchers.

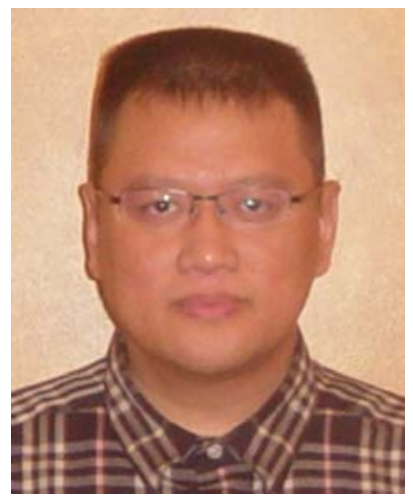

Dr. Yang Xiao worked in industry as a MAC (Medium Access Control) architect involving the IEEE 802.11 standard enhancement work before he joined Department of Computer Science at The University of Memphis in 2002. He is currently with Department of Computer Science at The University of Alabama. He was a voting member of IEEE 802.11 Working Group from 2001 to 2004. He is an IEEE Senior Member. He is a member of American Telemedicine Association. He currently serves as Editorin-Chief for International Journal of Security and Networks (IJSN), International Journal of Sensor Networks (IJSNet), and International Journal of Telemedicine and Applications (IJTA). He serves as a referee/ reviewer for many funding agencies, as well as a panelist for NSF and a member of Canada Foundation for Innovation (CFI)'s Telecommunications expert committee. He serves on TPC for more than 100 conferences such as INFOCOM, ICDCS, MOBIHOC, ICC, GLOBECOM, WCNC, etc. He serves as an associate editor for several journals, e.g., IEEE Transactions on Vehicular Technology. His research areas are security, telemedicine, sensor networks, and wireless networks. He has published more than 290 papers in major journals (more than 60 in various IEEE journals/magazines), refereed conference proceedings, book chapters related to these research areas. Dr. Xiao's research has been supported by the US National Science Foundation (NSF), U.S. Army Research, Fleet \& Industrial Supply Center San Diego (FISCSD), and The University of Alabama's Research Grants Committee.

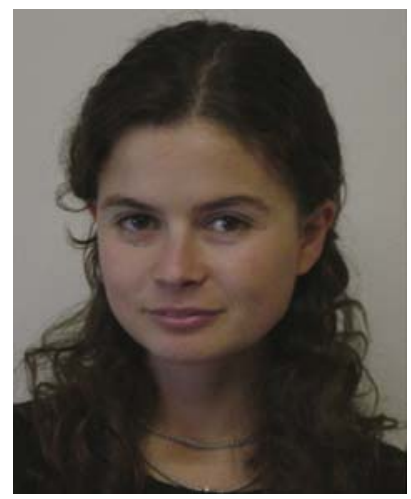

Ilenia Tinnirello has been Assistant Professor at the University of Palermo since January 2005. She received the Laurea degree in Electronic Engineering and the $\mathrm{Ph}$. D. on Communications, respectively in April 2000 and February 2004. She has also been Visiting Researcher at the Seoul National University, Korea, in 2004, and at the Nanyang Technological University of Singapore in 2006. Her research activity has been mainly focused on wireless networks and in particular on: multiple access algorithms with quality of service provisioning; cross-layer interactions between access solutions and physical layer; mobility management and load balancing in wireless packet networks.

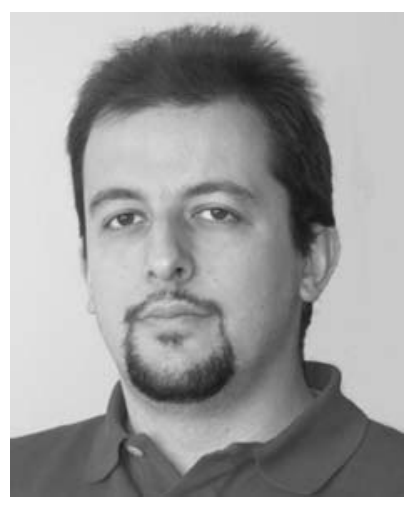

Fabrizio Granelli received the "Laurea" (M.Sc.) degree in Electronic Engineering from the University of Genoa, Italy, in 1997, with a thesis on video coding, awarded with the TELECOM Italy prize, and the Ph.D. in Telecommunications, in 2001. Since 2000 he is teaching as Assistant Professor in Telecommunications and Coordinator of the Networking Research Laboratory at the Dept. of Information Engineering and Computer Science-University of Trento (Italy). In August 2004, he was visiting professor at the State University of Campinas (Brasil). He is author or coauthor of more than 80 papers published in international journals, books and conferences, focused on wireless and mobile networks, performance evaluation and enhancement, multimedia communications. Dr. Granelli is Guest-Editor of 3 special issues of ACM Journal on Mobile Networks and Applications, focused on wireless networks, and Technical Program Co-Chair of the Globecom Symposium on Communications QoS, Reliability and Performance Modeling (2007-2009). He is General Vice-Chair of the First International Conference on Wireless Internet (WICON'05), General Chair of the 11th IEEE Workshop on ComputerAided Modeling, Analysis, and Design of Communication Links and Networks (CAMAD’06) and Technical Program Co-Chair of the 13th IEEE Workshop on Computer-Aided Modeling, Analysis, and Design of Communication Links and Networks (CAMAD'08). He is Senior Member of IEEE, Chair of the IEEE ComSoc Technical Committee on Communication Systems Integration and Modeling (CSIM) and Associate Editor of IEEE Communications Letters, IEEE Communications Surveys and Tutorials and International Journal on Communication Systems. 


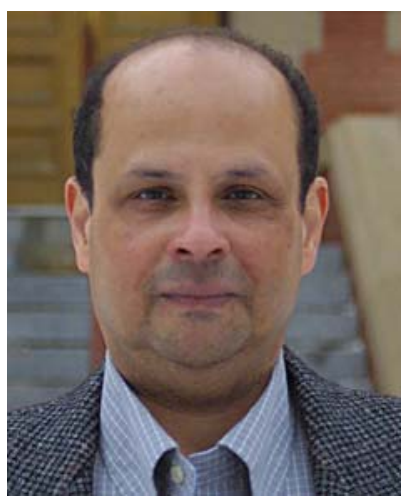

Ehab Elmallah is a Professor at the Department of Computing Science at the University of Alberta in the areas of broadband and wireless network architecture, resource management algorithms and protocols, control and performance evaluation, and combinatorial algorithms for scheduling and network reliability analysis. Dr. Elmallah obtained the Ph.D. and the M.Sc. in Computing Science from the University of Waterloo and the University of Saskatchewan, Canada, and the B. Sc. in Computer and Systems Engineering from Alexandria University, Egypt. He has numerous publications in reputable journals and conferences. He is a Foundation Fellow of the Institute of Combinatorics and its Applications and a Senior Member of the IEEE. Dr. Elmallah serves on the editorial boards of the International Journal of Sensor Networks and the International Journal of Network Management. He serves on the organizing and program committees of numerous international conferences. Currently, he is the General Chair of the 34th IEEE Conference on Local Computer Networks (LCN). 